\title{
Optical absorption and Photoluminescence studies in Gamma-irradiated nanocrystalline $\mathrm{CaF}_{2}$

\author{
C. Pandurangappa ${ }^{1 *}$ and B.N. Lakshminarasappa ${ }^{2}$
}

${ }^{1}$ Department of Physics, RNS Institute of Technology, Channasandra, Bangalore-560061, India

${ }^{2}$ Department of Physics, Jnanabharathi Campus, Bangalore University, Bangalore-560056, India

\begin{abstract}
Calcium fluoride $\left(\mathrm{CaF}_{2}\right)$ nanoparticles were synthesized by co-precipitation method and characterized by powder $X$-ray diffraction (PXRD), Fourier transform infrared (FTIR) spectroscopy, scanning electron microscopy (SEM). Also, optical absorption $(\mathrm{OA})$ and photoluminescence $(\mathrm{PL})$ studies on gamma irradiated ( $\mathrm{y}$-rayed) $\mathrm{CaF}_{2}$ nanoparticles were carried out. The XRD patterns confirmed the cubic crystallinity of the samples and the particle size was found to be $\sim 25 \mathrm{~nm}$. The purity of the synthesized nanoparticles was confirmed by FTIR spectrum. The morphological features studied using SEM revealed the agglomerated and porous nature of nanoparticles. $y$-rayed $\mathrm{CaF}_{2}$ nanoparticles showed a prominent absorption with a peak at $\sim 360 \mathrm{~nm}$ besides three weak but well separated absorptions at $\sim 267,442$ and $510 \mathrm{~nm}$. The various defect centers responsible for the absorption peaks were identified. The PL studies of samples showed strong emissions at $\sim 396 \mathrm{~nm}$ and $425 \mathrm{~nm}$. The observed PL emissions are attributed to defects created in nanocrystalline $\mathrm{CaF}_{2}$
\end{abstract}

Keywords: Synthesis; Nanoparticles; Scanning electron microscopy; Optical absorption; Photoluminescence

\section{Introduction}

The strong interest in nanotechnology stems from the concept that structures may be designed and built that exhibit superior electrical, mechanical, chemical or optical properties compared to the materials we know today. Some of the key issues in using nanoscale materials include the location, distribution of trapping centers, electron-phonon interactions and the potential for energy transfer to a luminescent center in a nanostructure. Many of the preparation methods of nanoparticles can be modified to create nanostructured films and nanocomposites, although some types of nanostructures require completely novel approaches [1-5].

Fluorides have attractive structural and optical characteristics. One of the fluorides with wide band gap and large scale transparency is Calcium fluoride $\left(\mathrm{CaF}_{2}\right)$. It is well established that when $\mathrm{CaF}_{2}$ crystals are irradiated with high energetic radiations such as Gamma, X-ray, elecrtron beam, ion beam they generate defects and give rise to color centers. These centers can be identified from various experimental techniques like OA, PL and TL. Literature reveals that irradiation effects on nanocrystalline $\mathrm{CaF}_{2}$ are limited. The defects studies in $\mathrm{CaF}_{2}$ nanocrystals are rare. Hence, the aim of the present work is to irradiate synthesized and characterized (XRD, FTIR, SEM) $\mathrm{CaF}_{2}$ nanoparticles by $\gamma$-rays and to study the defects created by OA and PL techniques.

\section{Experimental}

Calcium chloride $\left(\mathrm{CaCl}_{2}\right)$ and ammonium fluoride $\left(\mathrm{NH}_{4} \mathrm{~F}\right)$ were added into the conical flask in stoichiometric quantities and the mixture was dissolved in $100 \mathrm{ml}$ distilled water. The mixture was stirred constantly for 2 hours using a magnetic stirrer. The transparent reaction mixture transformed into opaque white suspension gradually. The stirred solution was centrifuged for 10 minutes at $5000 \mathrm{rpm}$ and a white residue was obtained. The residue was washed thoroughly with ethanol and the product was extracted on to a ceramic dish and dried slowly on a sand bath.

The structure of the synthesized samples was confirmed by XRD measurements using Philips X-pert PRO powder diffractometer with $\mathrm{Cu}-\mathrm{K}_{\alpha}$ radiation $(\lambda=1.54056 \AA)$ in the scan range $10-90^{\circ}$ at a low speed of $\sim 1^{\circ} /$ minute. The morphology of synthesized samples was studied using scanning electron microscopy (JEOL JSM-840A) by sputtering technique with gold as covering contrast material. The FTIR spectrum was recorded using Nicolet Magna 550 spectrometer with $\mathrm{KBr}$ pellets in the range $400-4000 \mathrm{~cm}^{-1}$. The samples were exposed to $\gamma$-rays from a $\mathrm{Co}^{60}$ source with an activity $3.89 \mathrm{KGy} / \mathrm{hr}$ for doses in the range $0.0324-$ $15.56 \mathrm{KGy}$. The Optical absorption measurements of the $\gamma$-rayed samples were carried out in the wavelength range 200-900 $\mathrm{nm}$ using V-570 UV/VIS/NIR double beam spectrophotometer by dispersing the samples in liquid paraffin (nuzol). The PL emission spectra of the $\gamma$-rayed samples was recorded at room temperature using a Fluorolog 3 Jobin Yvon spectrofluoremeter (FL3-11) equipped with a 450W Xenon lamp as the excitation source.

\section{Results and Discussion}

\section{PXRD, SEM and FTIR}

Figure 1 shows the PXRD pattern of nanocrystalline $\mathrm{CaF}_{2}$. The pattern reveals cubic phase of the fluorite type structure (JCPDS Card no. 87-0971) [6]. The displayed peaks correspond to $<\mathrm{h} \mathrm{k} \mathrm{l}>$ values $(1$ $\left.\begin{array}{ll}1 & 1\end{array}\right),\left(\begin{array}{lll}2 & 2 & 0\end{array}\right),\left(\begin{array}{lll}3 & 1 & 1\end{array}\right),\left(\begin{array}{lll}4 & 0 & 0\end{array}\right),\left(\begin{array}{lll}3 & 1 & 1\end{array}\right)$ and $\left(\begin{array}{lll}4 & 2 & 2\end{array}\right)$. The results match well with literature $[7,8]$.Using the $\langle\mathrm{h} \mathrm{k} \mathrm{l}>$ values the lattice constant (a) of the sample was calculated. The average value of lattice constant was found to be $\mathrm{a}=5.454 \AA$ which is in good agreement with literature value $\mathrm{a}=5.4355 \AA$ [9]. The XRD pattern presents broad peaks revealing the small crystallite size of the synthesized samples. The crystallite size was calculated from the full width at half maximum (FWHM) technique

${ }^{*}$ Corresponding author: Dr. C Pandu Rangappa, RNS Institute of Technology Bangalore, Karnataka, INDIA, E-mail: cpandu@gmail.com, cpandurangappa@ yahoo.com

Received January 04, 2011; Accepted February 19, 2011; Published February 23, 2011

Citation: Pandurangappa C, Lakshminarasappa BN (2011) Optical absorption and Photoluminescence studies in Gamma-irradiated nanocrystalline $\mathrm{CaF}_{2}$. J Nanomedic Nanotechnol 2:108. doi:10.4172/2157-7439.1000108

Copyright: (c) 2011 Pandurangappa C, et al. This is an open-access article distributed under the terms of the Creative Commons Attribution License, which permits unrestricted use, distribution, and reproduction in any medium, provided the original author and source are credited. 


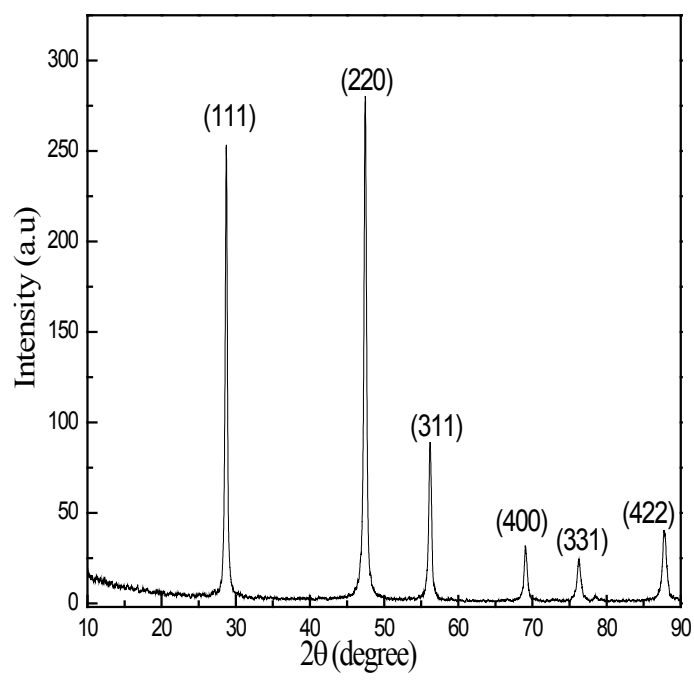

Figure 1: Powder XRD spectrum pure nanocrystalline $\mathrm{CaF}_{2}$.

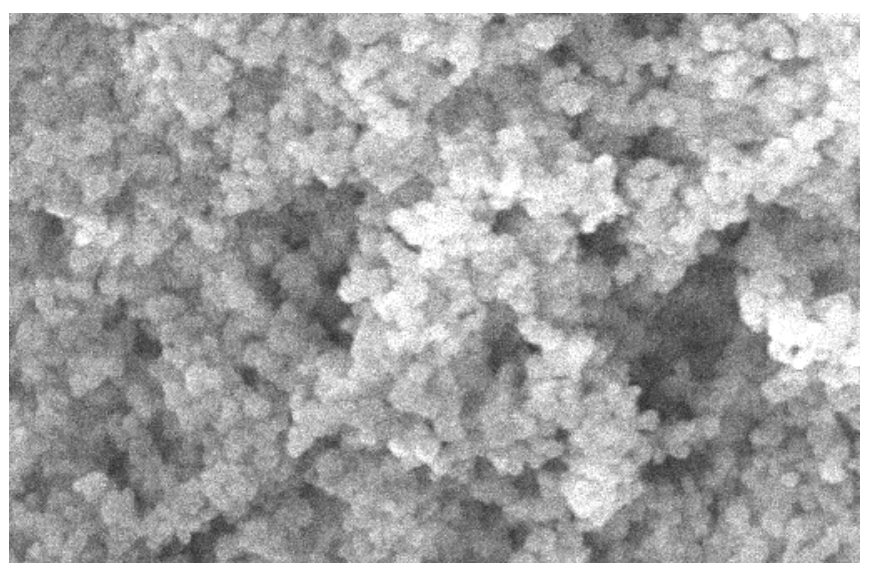

Figure 2: SEM pictures of pure nanocrystalline $\mathrm{CaF}_{2}$.

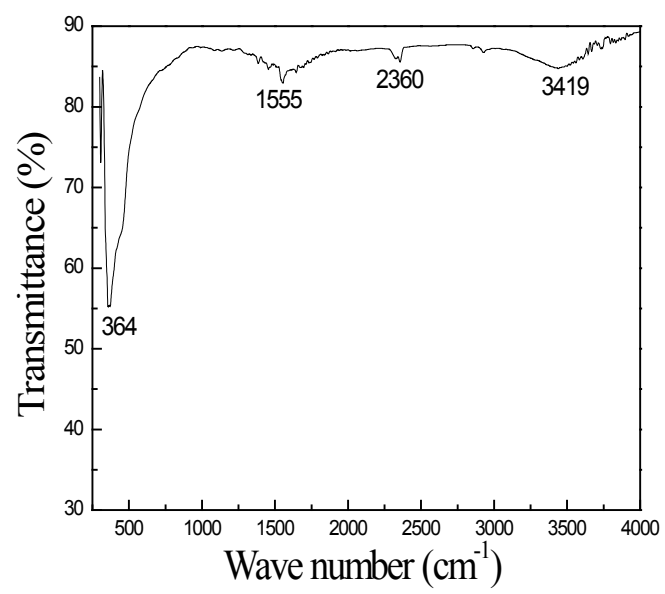

Figure 3: FTIR Spectra of pure nanocrystalline $\mathrm{CaF}_{2}$

using Scherer's formula $D=K \lambda /(\beta \cos \theta)$ where $K$ is the constant $(0.99)$, $\lambda$ is the wavelength of $\mathrm{Cu}-\mathrm{Ka}(1.54 \AA)$ line, $\beta$ is the FWHM and $\theta$ is the diffraction angle. The average crystallite size of nanoparticles was found to be $\sim 25 \mathrm{~nm}$. It is observed that the PXRD pattern of $500^{\circ} \mathrm{C}$ heat treated samples was similar to that of as prepared one but with slight increase in intensity. This indicates that $500^{\circ} \mathrm{C}$ heat treatment does not reveal any structural changes in the nanocrystalline $\mathrm{CaF}_{2}$. However, the crystallite size was found to be increased to $32 \mathrm{~nm}$. Thus heat treatment of the synthesized samples causes agglomeration of nanoparticles.

The SEM picture of as prepared nanocrystalline $\mathrm{CaF}_{2}$ is shown in Figure 2. The SEM results reveal the morphology of the synthesized nanoparticles. The as prepared nanopowder is agglomerated with polycrystalline nanoparticles. The agglomeration ranged from few microns to a few tens of microns. The SEM picture shows that $\mathrm{CaF}_{2}$ nanoparticles have many voids and are fluffy and porous. The larger particles exhibited numerous spherical perturbances on the surface, suggesting that they were formed during the precipitation process through fusion of the smaller particles.

FTIR spectrum was measured to check the purity of the synthesized powder. Figure 3 shows the FTIR spectrum of as prepared $\mathrm{CaF}_{2}$ nanoparticles. The spectrum shows two strong IR absorption bands at $\sim 3400$ and $1550 \mathrm{~cm}^{-1}$. They are characteristic of $\mathrm{H}-\mathrm{O}-\mathrm{H}$ bending of the $\mathrm{H}_{2} \mathrm{O}$ molecules. This reveals the presence hydroxyl groups in the as prepared sample [10]. The fundamental frequency at $\sim 364 \mathrm{~cm}^{-1}$ arises due to hindered rotations of the hydroxyl ions [11]. The band at $\sim 2357 \mathrm{~cm}^{-1}$ is due to $\mathrm{KBr}$ pellets used for recording FTIR spectrum. The FTIR pattern of $500^{\circ} \mathrm{C}$ heat treated samples showed decrease in the absorption of each band. The decrease in absorption of the 3419 and $1555 \mathrm{~cm}^{-1}$ bands on heating could be attributed to the release of water molecules trapped inside the solid matrix.

\section{Optical absorption studies}

The optical absorption spectrum of pristine and $\gamma$-rayed pure $\mathrm{CaF}_{2}$ nanocrystals is shown in Figure 4 . The pristine sample showed a weak absorption band at $\sim 370 \mathrm{~nm}$. The $\gamma$-rayed $\mathrm{CaF}_{2}$ nanocrystals showed a prominent absorption at $\sim 360 \mathrm{~nm}$ besides three weak ones at $\sim 267$, 442 and $510 \mathrm{~nm}$. The optical absorption increased marginally with increase in $\gamma$-dose, but the peak positions were unaltered with increase in $\gamma$-dose. The origine of the absorption bands can be explained in the following way. It is well established that nanoscale materials have large surface to volume ratio. This results in the formation of voids on the surface as well inside the agglomerated nanoparticles. Such voids can cause fundamental absorption in the UV wavelength range [12]. Also, surfaces of nanoparticles are well known to comprise of several defects

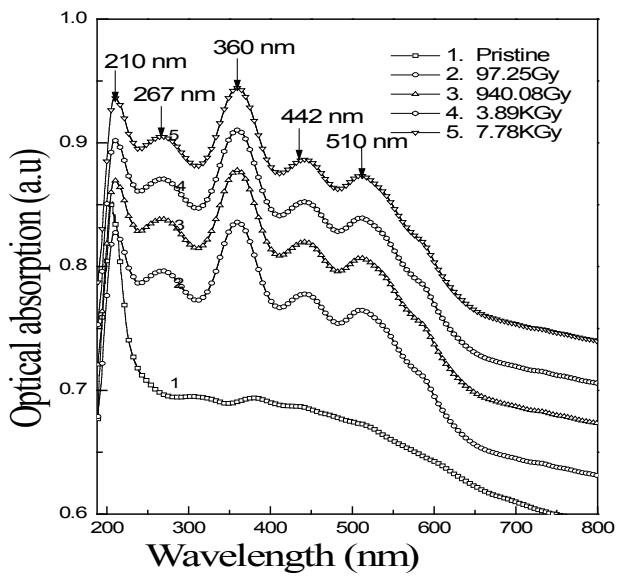

Figure 4: Optical absorption spectrum of $\mathrm{y}$-rayed pure nanocrystalline $\mathrm{CaF}_{2}$. 


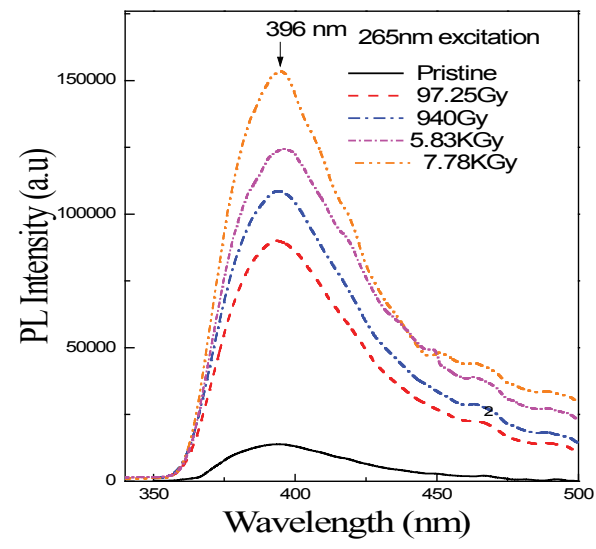

Figure 5: Photoluminescence spectrum of $\mathrm{y}$-rayed pure nanocrystalline $\mathrm{CaF}_{2}$ under $265 \mathrm{~nm}$ excitation.

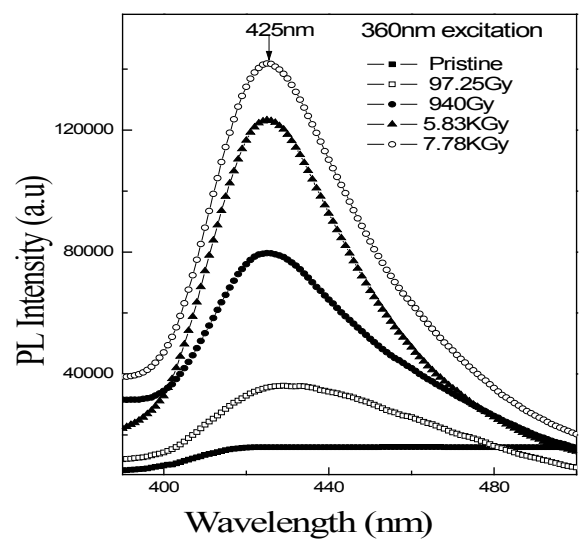

Figure 6: Photoluminescence spectrum of $\mathrm{y}$-rayed pure nanocrystalline $\mathrm{CaF}_{2}$ under $360 \mathrm{~nm}$ excitation.

such as Schottky or Frenkel which result in absorption of light by nanocrystals. Xiaoming Zhang et al. have reported similar absorption bands in $\mathrm{CaF}_{2}$ nanoparticles in the wavelength range $260-320 \mathrm{~nm}$ and attributed them to surface defects [13]. Thus the absorption bands at $267 \mathrm{~nm}$ in the present study may be attributed to surface defects such as Schottky or Frenkel in nanocrystalline $\mathrm{CaF}_{2}$.

It is well established that when $\mathrm{CaF}_{2}$ single crystals are $\gamma$-rayed F-centers are formed at room temperature. The F-center consists of an electron trapped at fluorine vacancy. -irradiation produces free electrons which when get trapped at negative ion vacancies form the F-centres. Literature reveals that irradiated $\mathrm{CaF}_{2}$ crystals exhibit the fundamental absorption at $375 \mathrm{~nm}$ and it is attributed to F-center [1416]. Considerable deviations in the coloration spectra are reported for samples studied by different workers depending upon the nature of impurities, method of preparation and other varying factors. Based on this discussion the absorption band at $360 \mathrm{~nm}$ in the present study is attributed to F-center generated during $\gamma$-irradiation.

During $\gamma$-irradiation, there will be diffusion of fluorine ions resulting in the fluorine vacancies. Some fluorine may occupy interstitial sites as neutral atoms or molecules. The creation of fluorine vacancies in calcium fluoride is accompanied by the formation of fluorine-rich defects such as $\mathrm{H}$ centers. The $\mathrm{H}$ center is a $\mathrm{F}_{2}$ ion occupying a fluorine lattice site. The absorption band of $\mathrm{H}$ center in $\mathrm{CaF}_{2}$ single crystals lies in the wavelength range 440-460 $\mathrm{nm}$ [17]. Hence, the $442 \mathrm{~nm}$ absorption band in the present case may be attributed to $\mathrm{H}$ center.

It is reported that at room temperature, the thermal diffusion and mobility of $F$ and $H$ centers in $\mathrm{CaF}_{2}$ promotes aggregation of nanoparticles, which consequently grow larger and absorb strongly at $520 \mathrm{~nm}$ [18]. Thus the band at $510 \mathrm{~nm}$ is attributed to Mie absorption of the nanoparticles [19]. These results indicate that defects are created in nanocrystalline $\mathrm{CaF}_{2}$ similar to that of bulk on their exposure to gamma radiations. However, the number of defects created and their response to $\gamma$-dose is different.

\section{Photoluminescence studies}

Figure 5 shows the $\mathrm{PL}$ emission spectrum of the $\mathrm{CaF}_{2}$ nanoparticles. A strong emission peak at $\sim 396 \mathrm{~nm}$ was observed when the samples were excited at $265 \mathrm{~nm}$. However, when the crystals were excited at 360 $\mathrm{nm}$ a strong emission was observed at $\sim 425 \mathrm{~nm}$ (Figure 6). The PL intensity increased with dose till 3.89 KGy and decreased with further increase in dose.

Major sources of luminescence in crystalline $\mathrm{CaF}_{2}$ are the selftrapped exciton (STE), F and F-aggregate centers created by irradiation. It is well established that irradiation of $\mathrm{CaF}_{2}$ leads to the formation of $\mathrm{F}$ and $\mathrm{F}$-aggregate centers in it. $\mathrm{F}-$ center has an absorption peak in the range $360-380 \mathrm{~nm}$. When the sample containing F-centers is excited with UV light, due to electronic transitions in the sample, emission of light takes place and the energy absorbed by the defects gets released in the form of photons leading to PL emission. The PL emission at 396 $\mathrm{nm}$ is attributed to F-center formed during $\gamma$-irradiation [17]. X. Zhang etal. observed similar emission at $\sim 400 \mathrm{~nm}$ in nanocrystalline $\mathrm{CaF}_{2}$ and attributed them to the presence of electronic centers [13].

Under $\gamma$-irradiation STE are typically formed when a free electron is localized at a self-trapped hole. The resulting excitations can decay radioactively yielding a distinctive luminescence [20]. Many $F-H$ center pairs produced by STE decay are promptly destroyed by recombination. The PL emission peak at $425 \mathrm{~nm}$ is attributed to surface defects like STE [21].

\section{Conclusions}

$\mathrm{CaF}_{2}$ nanoparticles were synthesized successfully by co-precipitation method and the average particle size was found to be in the range 25 $\mathrm{nm}$. The as prepared samples were agglomerated fluffy, porous and contain small traces water molecules. Heat treatment resulted in the release of water molecules and increase in particle size without any structural change. Optical absorption and Photoluminescence studies revealed the presence of number of defect centers in the $\gamma$-irradiated $\mathrm{CaF}_{2}$ nanoparticles.

\section{References}

1. Kenyon AJ (2002) Recent developments in rare-earth doped materials for optoelectronics. Prog in Quantum Electronics 26: 225-284.

2. Burda C, Chen XB, Narayanan R, El-Sayed MA (2005) Chemistry and properties of nanocrystals of different shapes. Chem Rev 105: 1025-1102.

3. Cushing BL, Kolesnichenko VL, O Connor CJ (2004) Recent advances in the liquid-phase syntheses of inorganic nanoparticles. Chem Rev 104: 3893-3946.

4. Luccas A, Debourg G, Jacquemet M, Druon F, Balembois F, et al. (2004) Moncorge High-power diode-pumped Yb3+: CaF2 femtosecond laser. Opt Lett 29: 2767-2769.

5. Zhou L, Chen D, Luo W, Wang Y, Yu Y, et al. (2007) Transparent glass ceramic containing $\mathrm{Er}^{3+}: \mathrm{CaF}_{2}$ nano-crystals prepared by sol-gel method. Materials Letters 61: 3988-3990. 
Citation: Pandurangappa C, Lakshminarasappa BN (2011) Optical absorption and Photoluminescence studies in Gamma-irradiated nanocrystalline $\mathrm{CaF}_{2}$. J Nanomedic Nanotechnol 2:108. doi:10.4172/2157-7439.1000108

6. Fujihara S, Kadota Y, Kimura T (2002) Role of Organic Additives in the Sol-Gel Synthesis of Porous $\mathrm{CaF}_{2}$ Anti-Reflective Coatings. JI of Sol-Gel Science and Technology 24: 147-154

7. Hong BC, Kawano K (2008) Reduction of $\mathrm{Eu}^{2+}$-activated nanoparticles by unique TCRA treatment Journal of Alloys and Compounds 451: 276-279.

8. Labeguerie J, Gredin P, Mortier M, Patriarche G, de Kozak A, et al. (2006) Synthesis of Fluoride Nanoparticles in Non-Aqueous Nanoreactors. Luminescence Study of $\mathrm{Eu}^{3+}: \mathrm{CaF}_{2}$ Allg. Chem. 632: 1538.

9. Sun $\mathrm{X}, \mathrm{LiU}(2003)$ Size-controllable luminescent single crystal $\mathrm{CaF}_{2}$ nanocubes. Chemical Communication 14: 1768-1769.

10. Huang $\mathrm{XH}$, Chen ZH (2005) Mater. Res Bull 40: 105.

11. Kenneth AW, Betty MH (1959) Physica 25: 569.

12. Kumar GA, Chen CW, Ballato J, Riman RE (2007) Optical Characterization of Infrared Emitting Rare-Earth-Doped Fluoride Nanocrystals and Their Transparent Nanocomposites. Chem Mater 19: 1523-1528.

13. Zhang X, Quan Z, Yang J, Yang P, Lian H et al. (2008) Solvothermal synthesis of well-dispersed $\mathrm{MF}_{2}(\mathrm{M}=\mathrm{Ca}, \mathrm{Sr}, \mathrm{Ba})$ nanocrystals and their optical properties. Nanotechnology 19: 075603.

14. Kamikawa T, Kazumata Y, Kikuchi A, Ozawa K (1966) The F center in calcium fluoride Physics Letters 21: 126-128.
15. David, Patterson, Robert G F (1967) F Band in X-and Electron-Irradiated $\mathrm{CaF}_{2}$ Physical Review Letters 18: 1123-1124.

16. Hayes W, Lambourn RF (1973) Production of F and F-Aggregate Centres in $\mathrm{CaF}_{2}$ and $\mathrm{SrF}_{2}$ by Irradiation. Phys Stat Sol 57: 693-699.

17. R Bennewitz, M Reichling, E Matthias (1997) Force microscopy of cleaved and electron-irradiated $\mathrm{CaF}_{2}(111)$ surfaces in ultra-high vacuum. Surf Sci 387: 6977 .

18. Cramer LP, Langford SC, Dickinson JT (2006) The formation of metallic nanoparticles in single crystal $\mathrm{CaF}_{2}$ under $157 \mathrm{~nm}$ excimer laser irradiation. $\mathrm{J}$ Appl Phys 99: 054305.

19. Cramer LP, Schubert BE, Petite PS, Langford SC, Dickinson JT (2005) Lase interactions with embedded $\mathrm{Ca}$ metal nanoparticles in single crystal $\mathrm{CaF}_{2} . \mathrm{J}$ Appl Phys 97: 074307.

20. Gorling C, Leinhos U, Mann K (2003) Self-trapped exciton luminescence and repetition rate dependence of two-photon absorption in $\mathrm{CaF}_{2}$ at $193 \mathrm{~nm}$. Opt Commn 216: 369-378

21. Xiang X, Zu XT, Zhu S, Ding TH, Wang LM (2006) Effects of electron irradiation and subsequent annealing on the optical absorption and photoluminescence of $\mathrm{CaF}_{2}$ single crystals. Optic Mat 28: 930-934. 